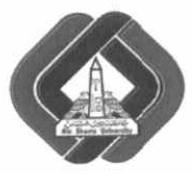

International Journal of Intelligent Computing and Information Sciences

\title{
ENHANCED FINGER VEIN BASED RECOGNITION SYSTEM
}

\author{
M. A. Jumaah
}

Faculty of Computers \& Information, Mansoura University, Egypt abdm1971@yahoo.com

\section{S. E. Hussein}

Faculty of Engineering, Mansoura University, Egypt sherif_hussein@mans.edu.eg
M. Z. Rashad

Faculty of Computers \& Information, Mansoura University, Egypt magdi_z2011@yahoo.com

Abstract: Robust Recognition systems become more complicated over time. These systems are derived from features which can be extracted from different body members using extractor methods. Finger vein is suitable member that could be used to violate the weakness of finger print. Conventional extractor methods like matched filter and morphological methods can extricate patterns if the widths of veins are steady whereas repeated line tracking method extract vein patterns from a hazy picture. These strategies can't remove veins that are smaller extensive than the accepted widths which corrupts the precision of the individual recognizable proof or can't adequately extricate flimsy veins on the grounds. In turn, we have proposed a system that tackles these issues by checking the shape of the picture profiles and stressing just the centerlines of veins. Our system for distinguishing the most extreme bend positions is hearty against transient vacillations in vein width and splendor. This paper introduces a finger vein recognition system based on using histogram of gradient and multi class support vector machine and finger vein recognition is powered by using Gabor filter with classifier powered by multi class support vector machine. The proposed have great enhancement impact over relative to accuracy, sensitivity, F-measure and precision during evaluation.

Keywords: Finger vein, Support Vector Machine SVM, Recognition System, Gabor, Histogram of Gradient HOG

\section{Introduction}

Recognition systems based on features extracted from different body members such as finger vein, print, and hand geometric, face, iris and much more. Those are used in identification systems based on biometric features. Personal identification technology is a most wide used systems for functions such as area access control and logins for PCs and e-commerce systems, the system start from sensing and acquire the data from external device which is responsible for detecting the details of the subject that participate and try to be recognized. Every image acquired from the capturing device is processed via sequential processes; pre-process, feature extraction and finally enrolling procedure and post processing at the against every subject enrolled in the database [1].

Recently, biometric recognition systems and their major usages are listed on top of the list. Many researchers' works on end [2]. Preprocessing phase includes aligning images, color mapping exchange and threshold detection. While, feature extraction methods are designed relative to each interested object and region of interest. These features are used to enroll details about he participated subject. These features are stored in the database repository which is accessed later for being compared against 
each recorded subjects for matching and recognitions process. In recognition phase, the classifier checks the feature vector of the test subject biometric fields, over recent year's research focus on improvements and developing recognition systems.

Yusuke Matsuda et. al [3] had proposed a finger-shape model and non-rigid registration method rather novel method for finger-vein authentication based on feature-point matching. Finger-vein images which contains artifacts such as irregular shading and vein posture deformation; because of capturing by using infrared light, can degrade accuracy due to irregular shading and vein deformation. Their proposed method, based on curvature of image-intensity profiles for feature point extraction, aimed to increase robustness. Asmaa et. al [4] had worked on operational difficulties of capturing images by using near infrared (NIR) illumination in conjunction with a camera of finger vein recognition systems. They worked on handling the mechanism of localizing the vein grid, and selecting the suitable feature vector to identify the vein pattern. Their proposed system had been based on Haar Wavelet Transform as feature extractor.

Yu Lu, Sook Yoon, Dong Sun Park [5] proposed a finger vein recognition innovation in view of scorelevel fusion matching of a solitary quality. To upgrade the nature of finger vein image, the contrastlimited adaptive histogram equalization (CLAHE) technique was used and it enhanced the nearby contrast of standardized image after ROI discovery. Gabor elements were then separated from eight channels in light of a bank of Gabor channels. Rather than utilizing the elements for the recognition specifically, they dissected the commitments of Gabor highlight from every channel and applied a weighted coordinating score-level fusion guideline to get the last coordinating score, which was utilized for the last recognition. Test results exhibited the CLAHE system was powerful to upgrade the finger vein picture quality and the proposed coordinating score level fusion demonstrated better recognition execution.

Lin et al [6] presented an algorithm for portioning the dorsal hand vein picture and separating the vein skeleton. After dim and measure normalizing, Gaussian low pass channel and middle channel were utilized to take out the bit commotion and the level strip filtering clamor separately. An enhanced NiBlack calculation portioned the vein design and a zone threshold calculation expelled the clamor obstructs from the vein design.

Jinfeng et al [7] concentrated on finger-vein upgrade and division in view of Gabor channels in the spatial area. Considering the high random city of the finger-vein arranges, a bank of even symmetric Gabor channels with eight introductions was used to adventure vein data in pictures. Picture remaking was executed to create a picture containing an incorporated finger-vein system. Naoto et al [8] proposed a technique for individual distinguishing proof in light of finger vein designs. A picture of a finger caught under infrared light contains the vein design as well as unpredictable shading created by the different thicknesses of the finger bones and muscles. The strategy removed the finger vein design from the misty picture by utilizing line following that began from different positions. Kuk et al [9] presented a finger-vein distinguishing proof framework based on a simple pattern-matching system for embedded applications that could accomplish invariant pattern matching with a set number of movements and pivots. This basic pattern-matching algorithm took into account the utilization of a cheap and quick equipment framework. Nancy et al [10] presented a proficient minutiae matching technique for finger vein acknowledgment. It included three stages minutiae pairing, false removing and matching. By utilizing morphological operations they had effectively taken out false minutiae to make distinguishing proof more exact. Matching was finished by chi square test to guarantee high exactness. Image retrieval was unreasonable and troublesome. 
Many researches were published to develop and enhance the accuracy of finger vein recognition systems based on uni-model, hybrid-model and fusion model. These systems had not optimized the recognition rate which needed to be enhanced. In the following paragraph, a finger vein recognition system was proposed that would utilize the performance over different evaluation metrics. The main goals of this paper is to introduce a finger vein recognition system based on using histogram of gradient and multi class support vector machine and another finger vein recognition is powered by using Gabor filter with classifier powered by multi class support vector machine and evaluation of the proposed strategy on recognition relative to accuracy, sensitivity, F-measure and precision.

\section{Methodology}

Biometric systems for recognizing people are shown in Fig. 1, which incorporate fingerprint, iris, and face recognition, are pulling in consideration in light of the fact that customary strategies, for example, keys, passwords, and PIN numbers convey the dangers of being forgotten, stolen, or lost [11].

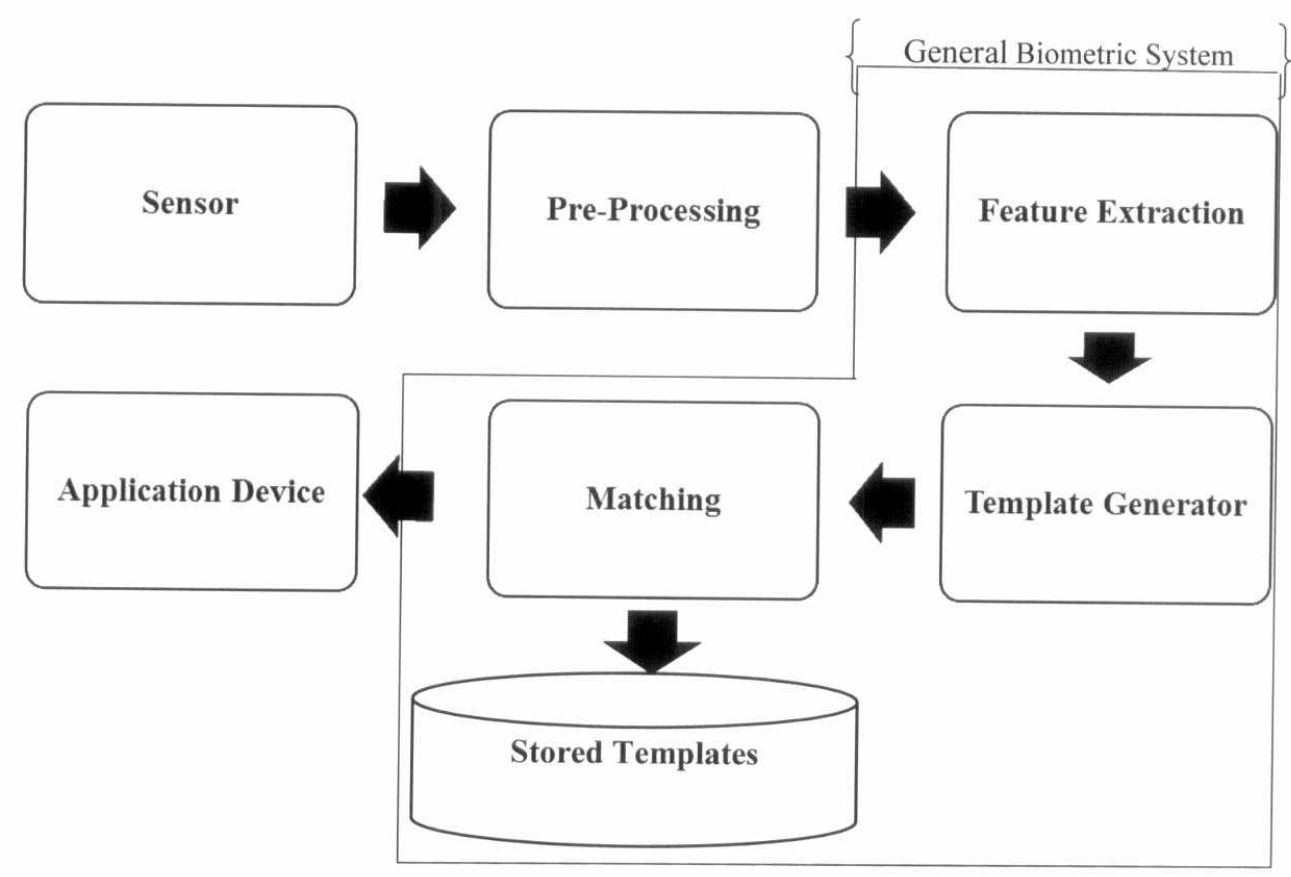

Figure 1. General Biometric Recognition System

Finger vein recognition framework is a biometric framework that uses finger-vein designs which being inside the human body, was beforehand proposed [Error! Reference source not found., 12]. This framework has the upside of being impervious to imitation. In this framework, infrared light is transmitted from the back of a hand. One finger is set between the infrared light source and a camera, as appeared in Fig. 2. As hemoglobin in the blood ingests infrared light, the finger vein examples are caught as shadow examples. The power of the light is balanced utilizing the caught picture. After that, 
the layout of the finger is distinguished, the turn of the picture is amended, and the vein example is extricated. At last, the example is contrasted and enrolled designs in a database [13].

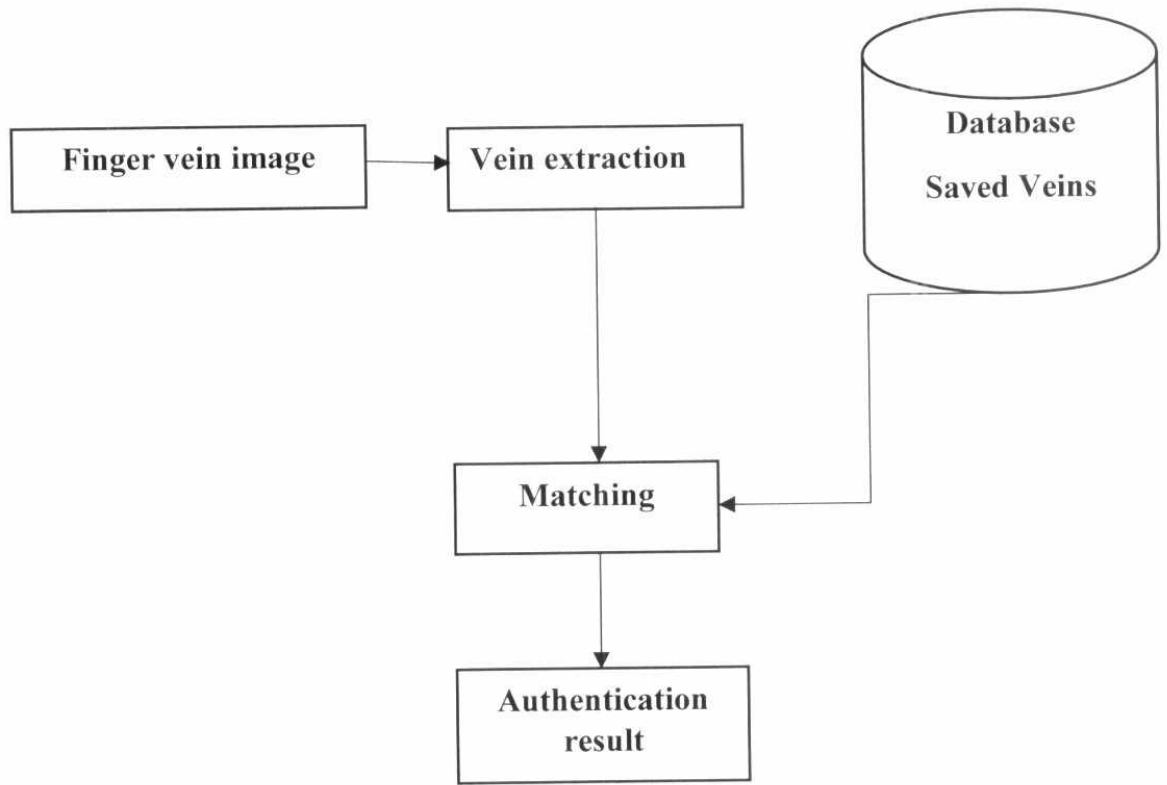

Figure. 2. Finger vein acquisition system [12]

A finger picture caught, utilizing infrared light, contains veins that have different widths and brightness, which might change with time as a result of vacillations in the measure of blood in the vein, brought on by changes in temperature, physical conditions, and so forth. To recognize a person with high exactness, the example of the thick and indistinct veins in a picture must be removed similarly. Besides, the example ought to be separated with almost no reliance on vein width and splendor variances [14].

Different common algorithms are used for processing image observed by their mapping strategies and allocation techniques. These algorithms are wide-ranging discussed because of their importance to derive the proposed system.

\subsection{Features extraction}

A system, for example, the matched filter and morphological strategies can extricate patterns if the widths of veins are steady. Then again, these routines can't extricate veins that are smaller/more extensive than the accepted widths, which debases the precision of the individual recognizable proof. The rehashed line following technique [2] can remove vein patterns from a hazy picture, yet it can't adequately separate flimsy veins on the grounds that the quantity of times that the following point proceeds onward thin veins has a tendency to be little measurably.

A method proposed by [17] is used here to solve these issues by checking the arch of the picture profiles and accentuating just the centerlines of veins. The centerlines are identified via hunting down positions where the arches of a cross-sectional profile of a vein picture are locally maximal. This technique for distinguishing the greatest shape positions is hearty against worldly variances in vein 
width and brilliance. The positions are joined with one another, and at long last the vein example is gotten.

$$
k(z)=\frac{d^{2} p_{f}(z) / d z^{2}}{\left\{1+\left(d p_{f}(z) / d z\right)^{2}\right\}^{\frac{3}{2}}}
$$

Where $F(x, y)$ is the intensity of $\operatorname{pixel}(x, y) \operatorname{Pf}(z)$ as a cross-sectional profile acquired from $F(x, y)$ at any direction and position, where $\mathrm{z}$ is a position in a profile. For instance, $\operatorname{Pf}(\mathrm{z})$ is acquired from $\mathrm{F}(\mathrm{x}, \mathrm{z})$ at a vertical direction.

\subsection{Global Threshold Selection}

It is important in picture processing to select an adequate threshold of gray level for extracting objects from their background. The histogram has a profound and sharp valley between two crests speaking to questions and foundation, individually, so that the limit can be picked at the base of this valley for most real pictures, it is frequently hard to identify the valley base accurately, particularly in such cases as when the valley is level and wide, pervaded with commotion, or when the two tops are to a great degree unequal in tallness, regularly delivering no traceable valley. The limit can be chosen at the dim level with the maximal measure of distinction. These use data concerning neighboring pixels (or edges) in the first picture to alter the histogram in order to make it helpful for threshold function. Another function works on directly sort the blurred level histogram by parametric strategies. For instance, the histogram is approximated at all square sense by a whole of Gaussian dispersions, and measurable choice methods are connected. Be that as it may, such a strategy requires extensively repetitive and sometimes unstable calculations. Besides, as a rule, the Gaussian circulations end up being a pitiful guess of the real modes the optimal threshold $\mathrm{k}^{*}$ is

Where $\sigma_{B}^{2}$ are functions of threshold level k.

$$
\sigma_{B}^{2}\left(k^{*}\right)=\max _{1 \subseteq k \leq L} \sigma_{B}^{2}(k)
$$

\subsection{Gabor Filters}

In image processing a Gabor filter, originally introduced by Dennis Gabor, is a linear filter used for edge detection. Frequency and orientation representations of Gabor filters are similar to those of the human visual system, and they have been found to be particularly appropriate for texture representation and discrimination. The one-dimensional Gabor filter is defined as the multiplications of a cosine as even function, see Fig 3.a or as the multiplication of sine as odd function, Fig 3.b. In Fig 3 each function is represented as a wave with Gaussian windows as follows [18]:

$$
\begin{aligned}
& g_{\theta}(x)=\frac{1}{\sqrt{2 \pi \sigma}} e^{-\frac{x^{2}}{2 \sigma^{2}}} \cos \left(2 \pi \omega_{0} x\right) \\
& g_{0}(x)=\frac{1}{\sqrt{2 \pi \sigma}} e^{-\frac{x^{2}}{2 \sigma^{2}}} \sin \left(2 \pi \omega_{0} x\right)
\end{aligned}
$$

Where $\omega_{0}$ defines the centre frequency (i.e., the frequency in which the filter yields the greatest response) and $\sigma$ the spread of the Gaussian window.

The power spectrum of the Gabor filter is given by the sum of two Gaussians centered at ${ }_{-}^{+} \omega_{0}$ :

Gabor filters is their invariance to rotation, scale, and translation. Furthermore, they are robust against photometric disturbances, such as illumination changes and image noise. The Gabor filter-based features are directly extracted from the gray-level images. In the spatial domain, a two-dimensional Gabor filter is a Gaussian kernel function modulated by a complex sinusoidal plane wave, defined as [19]: 


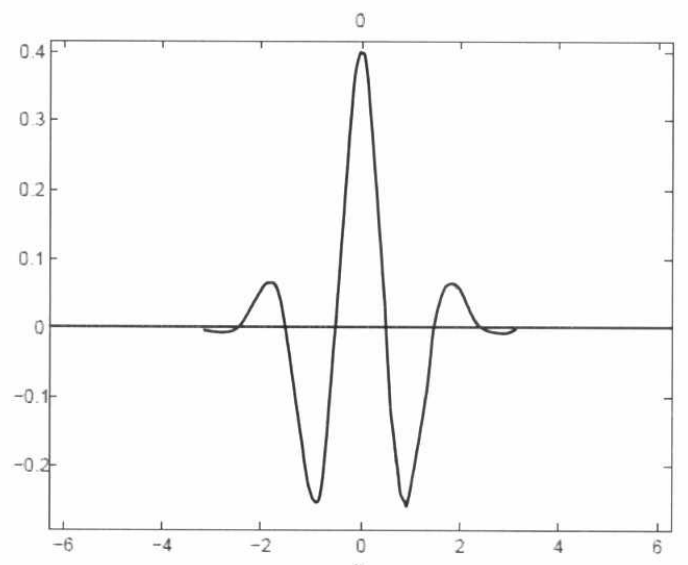

(a)

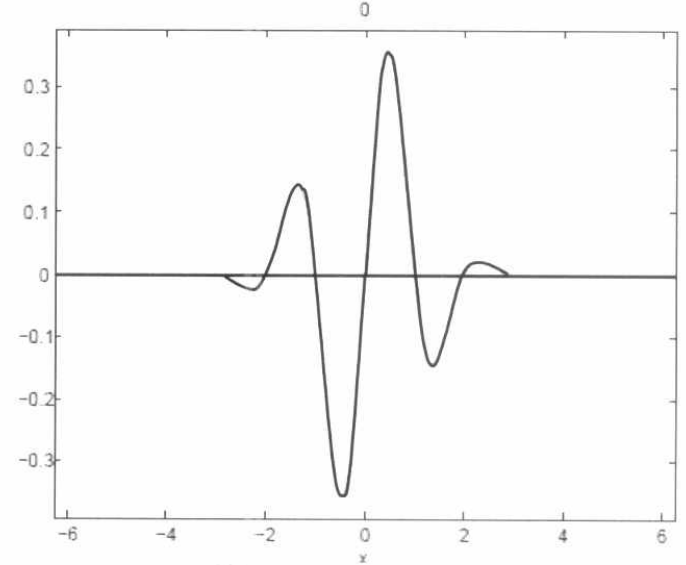

(b)

Figure. 3. Gabor cosine wave (a) even with a Gaussian window and Gabor sine wave (b) odd with a Gaussian window Gabor filters to extract features from the detected region. The most important advantage of

$$
\begin{aligned}
G(x, y) & =\frac{f^{2}}{\pi y \eta} \exp \left(-\frac{x^{\prime 2}+y^{2} y^{\prime 2}}{2 \sigma^{2}}\right) \exp \left(j 2 \pi f x^{\prime}+\emptyset\right) \\
x^{\prime} & =x \cos \theta+y \sin \theta \\
y^{\prime} & =-x \sin \theta+y \cos \theta
\end{aligned}
$$

Where $\mathrm{f}$ is the frequency of the sinusoid, h represents the orientation of the normal to the parallel stripes of a Gabor function, / is the phase offset, $r$ is the standard deviation of the Gaussian envelope and $\mathrm{c}$ is the spatial aspect ratio which specifies the elasticity of the support of the Gabor function [20].

\subsection{Histogram of Oriented Gradient}

The histogram of oriented gradients (HOG) is a component descriptor utilized as a part of PC vision and picture handling with the end goal of item identification. The strategy includes events of slope introduction restricted parts of a picture. This technique is like that of edge introduction histograms, scale-invariant component change descriptors, and shape connections, however varies in that it is processed on a thick network of consistently separated cells and utilizes covering nearby contrast standardization for enhanced exactness [26].

The basic idea is that local object appearance and shape can often be characterized rather well by the distribution of local intensity gradients or edge directions, even without precise knowledge of the corresponding gradient or edge positions [22, 23]. By partitioning the image window into little spatial districts "cells" for every cell amassing a neighborhood 1-D histogram of slope headings or edge introductions over the pixels of the cell. The consolidated histogram routes frame the representation in order to get better invariance, brightening, shadowing, and so on. It is additionally valuable to contrast standardize the nearby reactions before utilizing them $[24,25]$. This should be possible by aggregating a measure of nearby histogram "vitality" over to some degree bigger spatial areas (squares) and utilizing the outcomes to standardize the greater part of the cells in the piece. We will allude to the standardized descriptor hinders as Histogram of Oriented Gradient (HOG) descriptors. Tiling the identification 
window with a thick (truth be told, overlapping) framework of HOG descriptors and utilizing the joined element vector as a part of an ordinary SVM based window classifier gives our human discovery chain [26].

The Swine descriptor has a couple key focal points over different descriptors. Since it works on nearby cells, it is invariant to geometric and photometric changes, aside from item introduction. Such changes would just show up in bigger spatial locales. In addition, founded changes, coarse spatial inspecting, fine introduction testing, and solid neighborhood photometric standardization allows the individual body development of people on foot to be disregarded insofar as they keep up a generally upright position. The HOG descriptor is consequently especially suited for human identification in pictures. The Swine descriptor comprise of four stages as shown in Fig. ₹, which is commonly known as HOG descriptor [26].

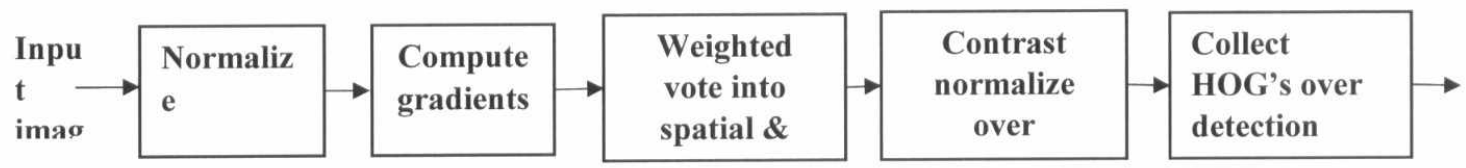

Figure. 4. The four stages to HOG descriptor for human identification in

The first stage of calculation in many feature detectors in image pre-processing is to ensure normalized color and gamma values (Gradient computation). The most common method is to apply the 1-D centered, point discrete derivative mask in one or both of the horizontal and vertical directions. Specifically, this method requires filtering the color or intensity data of the image with the following filter kernels [26]:

$$
D_{X}=\left[\begin{array}{lll}
-1 & 0 & 1
\end{array}\right] \text { and } D_{Y}=\left[\begin{array}{r}
1 \\
0 \\
-1
\end{array}\right]
$$

So, being given an image $I, \mathrm{x}$ and $\mathrm{y}$ derivation can be obtained using a convolution operation: $I_{x}=I * D_{x}$ and $I_{y}=I * D_{y}$

The magnitude of the gradient is $|G|=\sqrt{I_{x}^{2}+I_{y}^{2}}$

And the orientation of the gradient is given by: $\theta=\arctan \frac{L_{\underline{x}}}{L_{y}}$

The second stage of estimation is making the cell histograms (Orientation binning). Every pixel inside of the cell makes a weighted choice for an introduction construct histogram channel based with respect to the qualities found in the inclination calculation. The cells themselves can either be rectangular or outspread fit as a fiddle, and the histogram channels are uniformly spread more than 0 to 180 degrees or 0 to 360 degrees, contingent upon whether the slope is "unsigned" or "signed". Geometric and photometric changes found that unsigned inclinations utilized as a part of conjunction with 9 histograms directs performed best in their human recognition tests. Concerning the vote weight, pixel commitment can either be the inclination extent itself, or some capacity of the greatness. In tests, the inclination greatness itself for the most part delivers the best results. Different alternatives for the vote weight could incorporate the square root or square of the slope greatness, or some cut form of the size [22, 25].

The third stage is to represent changes in light and contrast (Descriptor obstructs), the slope qualities must be privately standardized, which requires gathering the phones together into bigger, spatially joined squares. The HOG descriptor is then the linked vector of the parts of the standardized cell histograms from the greater part of the square areas. These pieces commonly cover, implying that every cell contributes more than once to the last descriptor. Two fundamental piece geometries exist: 
rectangular R-HOG squares and round C-HOG squares. R-HOG pieces are for the most part square networks, spoke to by three parameters: the quantity of cells per hinder, the quantity of pixels per cell, and the quantity of channels per cell histogram.

The fourth stage is block normalization. There are different methods for block normalization. Let $v$ be non-normalized vector containing all histogram in given block, $\|v k\|$ be its k-norm for $\mathrm{k}=1,2$ and e be some small constant (whose value will not influence the results). Then the normalization factor can be one of the following [26]:

\section{Proposed Algorithm}

$$
\begin{aligned}
& \text { L2- norm: } f=\frac{v}{\sqrt{\|v\|_{2}^{2}+s^{2}}} \\
& L 1-\text { norm: } f=\frac{v}{\|v\|_{1}+\theta} \\
& L 1-\text { sqrt: } f=\sqrt{\frac{v}{\|v\|_{1}+\theta}}
\end{aligned}
$$

Because of the recognition system is based on identifying different subjects that are participated in the system so every subject represent a separate class which is considered independent class labels so that the classifier would be used must be a multi-label classifier that can recognize and identify the subject. SVM is used for such purposes that support multi-class. On other hand, the proposed system is powered by two different feature extraction mechanisms that lead to the contribution of the proposed system. The proposed system is based on using Gabor filter as feature extraction and SVM as classifier whereas, other system is based on using modified HOG as feature extraction and SVM as classifier.

\subsection{Preprocessing}

First, data is prepared for being processed through sequential subsequent phases. First for every image per subject is loaded in the gray scale mode. Next, vein image is adjusted using a threshold detected adaptive for each image. The vein image is segmented to recognize the region of interest, vein region. For the vein region a feature extractor is used to extract the most power features exists. These features are stored labeled for the subject name for father classification and recognition.

\subsection{Dataset Splitter}

The Dataset stacking stage is the procedure in charge of dividing the dataset into two sections got from the preprocessing module. Holdout system is utilized to part the dataset into two sections where given information are haphazardly divided into two autonomous sets, a preparation set and a test set. The preparation set is utilized to prepare the proposed framework while the test set is utilized to test and approve the exactness of the prepared model.

\subsection{Subject Enrollment Phase}

Subject enrollment is a basic process for inserting every new participate member into the recognition system. The process of enrollment is divided into sets of phases that are going in a sequence flow start from preprocessing mentioned in previous words followed by detecting the region of interest represented in a single finger vein boundary. The vein region is the input for feature extraction phase. Feature extraction of the proposed recognition system is dual mode. First mode is based on Histogram of Gradient filter (HOG) while the second mode is based on Gabor filter. 


\subsection{First mode}

In which a filter is designed based on a moveable window that is playing important role of quantity and quality of the features extracted from the vein shape. In finger vein of any person, the vein contains thick sharp lines in horizontal and vertical direction which represents constrains over the process of feature extraction. In the used dataset, vein image is directed in horizontal direction that leads to modify the window of the HOG filter to be adaptive over the vein lines. The window of the filter start scans from left to right at the top towards bottom. The window used for extracting features based on mining the rectangle region bounded by the window circumstance. The set of features is adaptive incremental to get all over the features of the vein for being vectored. HOG is effective feature extraction mechanism for getting feature in gray level color schema based on gradient operator. HOG is proposed by using a rectangular cell moving over the pixels of image regardless of direction or histogram of the image. The proposed modification of the HOG cell leads to gain the pros of both ordinary HOG approach besides tracking line feature that track the direction of line. That is achieved by dedicating the vein direction in horizontal followed by a directed window.

\subsection{Second mode}

On other hand of the proposed recognition system, another feature extraction is modified and optimized to be used known as Gabor filter. In Gabor filter, features are extracted from gray level images based on free of locations and orientation. In order to increase the precision and accuracy of the extracted feature from the vein, region of interest is extracted and directed horizontally. The modified mechanism is based on kernel function called Gaussian which is modulated by a complex sinusoidal plane wave. The feature is transformed to be stored as a vector.

\subsection{Database features}

Next of feature extraction phase using either HOG or Gabor filter, the feature vector of the subject is stored in the database for further comparison. The stored database of feature is used later for running model classification process that focus on construction of the classifier. The classifier is powered by the support vector machine, SVM, which represents the second generation of the neural networks mechanisms and structures. The model is built over three distinct separate phases; training, testing and validation.

\subsection{Testing Phase}

In this stage, the proposed framework utilizes the testing dataset which is produced at the Data Splitting module. Testing dataset is utilized as info for assessment the execution of the model. The results of this module is further sent to develop the aggregate classifier execution assessment stage that is talked about at the test area in points of interest utilizing the arrangement decides that were produced in Learning Phase and put away for the execution assessment of the proposed half breed interruption location framework.

\section{Results and Discussion}

The proposed framework was implanted and developed using MatLab 2014b x64 bit on Computer system with specific features. These features were classified as Hardware including Intel Processor, 2.0 $\mathrm{GHz}$ and 64 bit architecture rather than Windows 7 Home Edition as Software platform. The proposed system is based on using the dataset, vein data, which is available online for free in image format [27]. 
The Classifier Performance Evaluator carries out various performance measures of classification to evaluate the proposed framework. These measures are based on some principles includes, Recall, Specificity, Precision, Accuracy, G-mean and F-measure that can be defined as follows [28]:

- Recall or true positive rate measures the proportion of positives that are correctly identified.

- Specificity or true negative rate measures the proportion of negatives that are correctly identified.

- Precision is a description of a level of measurement that yields consistent results when repeated. It is associated with the concept of "random error", a form of observational error that leads to measurable values being inconsistent when repeated.

- Accuracy is a level of measurement with no inherent limitation (ie. free of systemic error, another form of observational error.

- GMEAN like mean, except that it skips over bad point.

- F-measure is one of the most commonly used "single number" measures in Information Retrieval, Natural Language Processing and Machine Learning, but it is based on a mistake is a weighted harmonic mean of Recall (Sensitivity)\& Precision (R \& P) [29].

The proposed system is evaluated using two of scenarios that are applied during execution of the experiments including using official online dataset. The two different scenarios are applied on the dataset recording different performance metric values; tablel shortened the two different scenarios that were used for experiment of the proposed system.

Table 1. Abbreviation of Evaluation Scenario for Experimental

\begin{tabular}{|l|l|}
\hline \multicolumn{1}{|c|}{ Scenario \# } & \multicolumn{1}{c|}{ Abbreviation } \\
\hline $\begin{array}{l}\text { Scenario [1] } \\
\text { "SC_1" }\end{array}$ & Perform feature extraction using HOG tailed with a SVM recognition strategy. \\
\hline $\begin{array}{l}\text { Scenario [2] } \\
\text { "SC_2" }\end{array}$ & Perform feature extraction using Gabor Filter tailed with a SVM recognition strategy. \\
\hline
\end{tabular}

First experiment was performed and denoted as "SC 1 " as short term for scenario 1. In which, the proposed system follows the first path of feature extraction based on Histogram of Gradient and powered by the SVM classification model for recognition purposes. Second scenario, "SC_2", is based on employing Gabor filter as feature extraction mechanism while the SVM still the classifier for recognition. The proposed system is evaluated using different performance metric that is responsible for testing recognition rate and system robustness .Table 2, represents the system recognition rate, accuracy, over the different phase of experiments. In the proposed system regards to the growth of learning and consciously recognition accuracy increased from $88.33 \%$ in both scenarios at validation phase to be $95 \%$ and $96.67 \%$ with high jump improvement interval at scenario 1 and scenario 2 respectively.

Table 2. Experimental Evaluation against Accuracy metric

\begin{tabular}{|c|c|c|}
\hline Scenario \# & Validation & Testing \\
\hline SC_1 & $88.33 \%$ & $95.00 \%$ \\
\hline
\end{tabular}




$$
\begin{array}{l|l|l|}
\text { SC_2 } & 88.33 \% & 96.67 \% \\
\hline
\end{array}
$$

Other metric is information retrieval measure that is related to rate of retrieving and recognize the correct subject participate due to F-measure. Regards to table 3, at the first scenario the information retrieval is enhanced from $46.15 \%$ to reach $66.67 \%$ to score $44.46 \%$ improvements. While at the second scenario, the proposed system enhances the information retrieval by $100 \%$ as it scores $75 \%$ instead of $37.5 \%$ at validation.

Table 3. Experimental Evaluation against F-measure metric

\begin{tabular}{|c|c|c|}
\hline Scenario \# & Validation & Testing \\
\hline SC_1 & $46.15 \%$ & $66.67 \%$ \\
\hline SC 2 & $37.50 \%$ & $75.00 \%$ \\
\hline
\end{tabular}

Because of the proposed system is measured in random mode, precision is tested to restrict the evaluation of the proposed system that yields great ad value scoring $50 \%$ and $60 \%$ at scenario 1 and scenario 2 instead of 0.03 and $23.08 \%$ respectively. See table 4 .

Table 4. Experimental Evaluation against Precision metric

\begin{tabular}{|c|c|c|}
\hline Scenario \# & Validation & Testing \\
\hline SC_1 & $0.30 \%$ & $50.00 \%$ \\
\hline SC_2 & $23.08 \%$ & $60.00 \%$ \\
\hline
\end{tabular}

Table 5 and table 6 conclude all different performance metrics that were discussed plus recall, G-mean and Specificity which were used to evaluate the proposed system over the two different phases. In table 5 , the proposed system have been validated which have improved over all of the proposed regards to different metric. In table 6 , the proposed system have been tested using same performance metric in which figure the proposed system keeping tracks of recall metric which is similar to recall listed in table 5 .

Table 5. Evaluation of validation Process against different metric

\begin{tabular}{|c|c|c|c|c|c|c|}
\hline Validation & Accuracy & Specificity & Precision & Recall & F-measure & Gmean \\
\hline SC_1 & $88.33 \%$ & $87.72 \%$ & $0.30 \%$ & $100.00 \%$ & $46.15 \%$ & $93.66 \%$ \\
\hline SC_2 & $83.33 \%$ & $82.46 \%$ & $23.08 \%$ & $100.00 \%$ & $37.50 \%$ & $90.81 \%$ \\
\hline
\end{tabular}

Table 6. Evaluation of testing Process against different metric

\begin{tabular}{|c|c|c|c|c|c|c|}
\hline Testing & Accuracy & Specificity & Precision & Recall & F-measure & Gmean \\
\hline SC_1 & $95.00 \%$ & $94.74 \%$ & $50.00 \%$ & $100.00 \%$ & $66.67 \%$ & $97.33 \%$ \\
\hline SC _2 & $96.67 \%$ & $96.49 \%$ & $60.00 \%$ & $100.00 \%$ & $75.00 \%$ & $98.23 \%$ \\
\hline
\end{tabular}


$100.00 \%$

$80.00 \%$
$60.00 \%$
$40.00 \%$
$20.00 \%$
$0.00 \%$

$.00 \%$

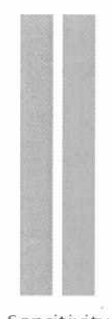

Sensitivity

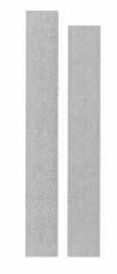

Specificity

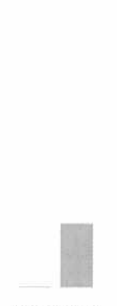

Precision

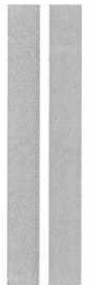

Recall

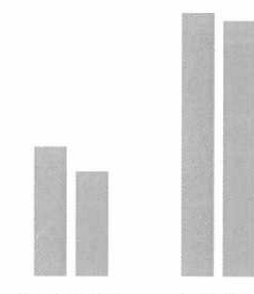

F-measure Gmean

Figure 5 Experimental Evaluation against different performance metric of the proposed system during validation phase

In Fig. 7 . The proposed have achieved high rates across all over performance metric except the recall that keep track of full level over two phase of experimental rather than shown in Fig. ${ }^{\circ}$. The proposed system achieved higher rates over more of training and learning iterations. The proposed system is based on free measurements of histograms and orientations rather except the vein direction should be horizontal for being used by HOG. While increasing the system learning procedures the system is achieving more recognition rate and robustness.

Testing of the proposed methods

$120.00 \%$
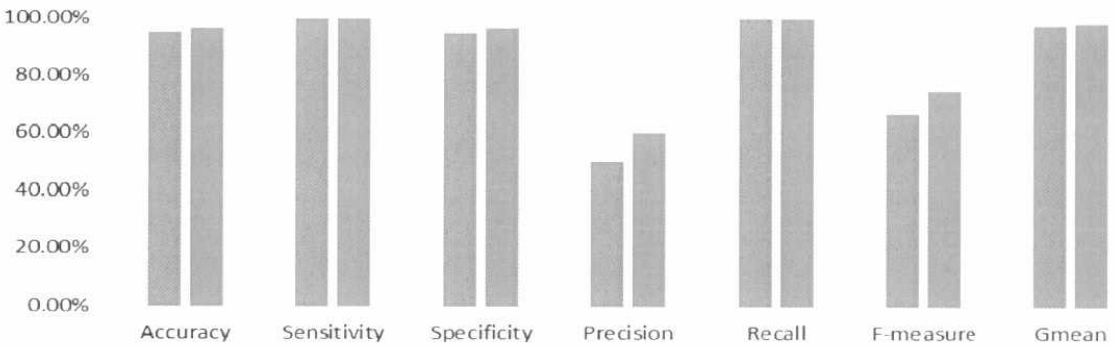

$=\mathrm{SC}_{-} 1=\mathrm{SC}_{-} 2$

Figure 6 Experimental Evaluation against different performance metric of the proposed system during testing phase

\section{Conclusion}

We have proposed and developed a finger vein recognition system based on histogram of gradient and multi class support vector machine and finger vein recognition powered by using Gabor filter with classifier powered by multi class support vector machine. The proposed system was evaluated using various performance procedures using available online dataset in image format. The proposed system scores accuracy variation from $88.33 \%$ in both scenarios at validation phase to be $95 \%$ and $96.67 \%$ with high jump improvement interval due to F-measure that is enhanced from $46.15 \%$ to $66.67 \%$ to score $44.46 \%$ improvements and $100 \%$ when it scores $75 \%$ instead of $37.5 \%$ at validation. Besides precision 
that yields great ad value scoring $50 \%$ and $60 \%$ instead of 0.03 and $23.08 \%$ during testing and validation.

\section{References}

1. R. L. D. T. Beining Huang, Yanggang Dai and W. Li, "Finger-vein authentication based on wide line detector and pattern normalization," in IEEE ICPR (I. ICPR, ed.), 2010.

2. H. U. M. Kono and S. Umemura, "A new method for the identifi cation of individuals by using of vein pattern matching of a finger," in in Proceedings of the Fifth Symposium on Pattern Measurement (Yamaguchi, ed.), (Japanese), p. 9-12, 2000.

3. Y. Matsuda, N. Miura, A. Nagasaka, H. Kiyomizu, and T. Miyatake, "Finger-vein authentication based on deformation-tolerant feature-point matching," Machine Vision and Applications, pp. 1-14, 2016.

4. R. E. F. Asmaa Q. Shareef, Loay E. George, "Finger vein recognition using haar wavelet transform," International Journal of Computer Science and Mobile Computing, vol. 4, pp. 1-7, March 2015.

5. D. S. P. Yu Lu, Sook Yoon, "Finger vein recognition based on matching score-level fusion of gabor features,” KICS, vol. 38A, no. 2, pp. 174-182, 2013.

6. X. L. Lin Yang and Z. Liu, "A skeleton extracting algorithm for dorsal hand vein pattern," in 2010 International Conference on Computer Application and System Modeling (ICCASM 2010) (ICCASM, ed.), vol. 22, pp. V13-92-V13-95, Oct 2010.

7. J. Y. Jinfeng Yang and Y. Shi, "Finger-vein segmentation based on multi-channel even-symmetric gabor filters," in IEEE International Conference on Intelligent Computing and Intelligent Systems 2009 (I. 2009, ed.), vol. 4 of 500-503, Nov 2009.

8. [A. N. Naoto Miura and T. Miyatake, "Feature extraction of finger-vein patterns based on repeated line tracking and its application to personal identification," Machine Vision and Applications, vol. 15, pp. 194-20, 2004.

9. M. A. Kuk Won Ko, Jiyeon Lee and S. Lee, "Development of human identification system based on simple finger-vein pattern-matching method for embedded environments," International Journal of Security and Its Applications IJSIA, vol. 9, no. 5, pp. 297-306, 2015.

10. N. Mantrao and S. Kaur, "An efficient minutiae matching method for finger vein recognition," International Journal of Advanced Research in Computer Science and Software Engineering IJARCSSE, vol. 5, pp. 657-660, June 2015.

11. T. Chkravorty, "Low cost subcutaneous vein detection system using arm9 single board computer," tech. rep., Department of Instrumentation and Control, 2011.

12. A. N. N. Miura and T. Miyatake, "Feature extraction of fi nger-vein patterns based on repeated line tracking and its application to personal identifi cation," Machine Vision and Applications, vol. 15, no. 4, p. 194-203, 2004.

13. R. Sujata Kulkarni, "Finger vein recognition," IOSR Journal of Electrical and Electronics Engineering (IOSR-JEEE), pp. 32-36, 2014.

14. A. K. . Y. Zhou, "Human identification using finger images," IEEE Transactions on Image Processing, vol. 21, pp. 2228-2244, April 2012.

15. V. K. A. Hoover and M. Goldbaum, "Locating blood vessels in retinal image by piece-wise threshold probing of a matched filter response," in IEEE Transactions on Medical Imaging (IEEE, ed.), 2000 . 
16. P. M. T. Walter, J. Klein and F. Zana, "Automatic segmentation and registration of retinal fluorescein angiographies application to diabetic retinopathy," in First InternationalWorkshop on Computer Assisted Fundus Image Analysis (Copenhagen, ed.), p. 15-20, May 2000.

17. A. N. N. Miura and T. Miyatake, "Extraction of finger-vein patterns using maximum curvature points in image profiles," in IAPR Conference on Machine VIsion Applications (T. S. City, ed.), vol. 8, pp. 347-350, May 2005.

18. G. D., "Theory of communication," Journal of the Institute of Electrical Engineers, vol. 93, p. 429457, 1946.

19. J. Y. . Y. Shi, "Finger vein based enhancement and segmentation," Springer, 2013.

20. K. G. Derpanis, "Gabor filters," tech. rep., York University, 2007.

21. B. T. N. Dalal, "Histograms of orientated gradients for human de," in IEEE Computer Society Conference on computer Vision and Pattern Recognition, 2005.

22. J. O. W. T. Freeman, K. Tanaka and K. Kyuma, "Computer vision for computer games," in 2nd International Conference on Automatic Face and Gesture Recognition (U. Killington, VT, ed.), pp. 100-105, October 1996.

23. W. T. Freeman and M. Roth, "Orientation histograms for hand gesture recognition," Intl. Workshop on Automatic Face and Gesture Recognition, IEEE Computer Society, pp. 296-301, June 1995.

24. K. Mikolajczyk and C. Schmid, "Scale and affine invariant interest point detectors," IJCV,, vol. 60, no. 1, pp. 63-86, 2004.

25. D. G. Lowe, "Distinctive image features from scale-invariant keypoints," IJCV, vol. 60, no. 2 , pp. $91-110,2004$.

26. N. Dalal and B. Triggs, "Histograms of oriented gradients for human detection," in IEEE Computer Society Conference on Computer Vision and Pattern Recognition, vol. 1, pp. 886-893, June 2005.

27. S. A. S. Mohd Shahrimie Mohd Asaari and B. A. Rosdi, "Fusion of band limited phase only correlation and width centroid contour distance for finger based biometrics," Expert Systems with Applications Elsevier, vol. 41, p. 3367-3382, June 2014. http://blog.eng.usm.my/fendi/?page id=262.

28. N. E. M. Mohamed A. Kassem and R. M. EL-Awady, "An enhanced atm security system using multimodal biometric strategy," International Journal of Electrical \& Computer Sciences IJECSIJENS, vol. 14, pp. 9-16, aug 2014.

29. D. M. Powers, "What the f--measure doesn't measure," tech. rep., Beijing University of Technology, Computer Science, Engineering \& Mathematics, Flinders University, 2014. 